\title{
Acquired vulvar lymphangioma circumscriptum: A report of 3 cases
}

\author{
Siham Mansouri, Sara Mai, Nadia Ismaili, Laila Benzekri, Badr Hassam \\ Department of Dermatology, Department of Dermatology, University Hospital Ibn Sina Rabat, Morocco
}

Corresponding author: Dr. Siham Mansouri, E-mail: siham-mnsr@hotmail.fr

\begin{abstract}
Vulvar lymphangioma circumscriptum (LC) is an unusual benign condition, congenital or acquired, related to interference in the lymph drainage. This entity can be confused with conditions such as genital warts. We sought to clarify the clinicopathologic features of the Acquired vulvar LC by studying 3 affected patients from our institution. We report 3 cases of vulvar LC: Two cases were presented after gynecologic cancer treatment (surgery, lymphadenectomy, and radiotherapy). 1 case was related to lower limb chronic lymphedema. In two of them, the initial clinical diagnosis was genital wart. Two patients were treated by Laser CO2 and cryotherapy, with a good response and without any recurrence. The possibility of vulvar LC should be taken into account as a possible diagnosis in patients with previous oncological surgery or genital warts refractory to the conventional treatment. Knowledge on the features of this lesion can avoid unnecessary clinical and therapeutic procedures.
\end{abstract}

Key words: Acquired; Vulvar; Lymphangioma circumscriptum

\section{INTRODUCTION}

Lymphangioma circumscriptum (LC) is a rare vascular malformation that affects the lymph vessels of the papillary dermis. LC can be either congenital or acquired after damaging the previously normal lymph channels [1]. It frequently appears in the armpit, the neck, and the proximal portion of the extremities [2]. Acquired vulvar LC (AVLC), is a rare, benign condition [3]. Most of the descriptions refer to isolated cases or short series of patients. We report 3 cases and describe the clinical and pathologic characteristics, to add to the limited data present in the literature.

\section{CASE REPORTS}

We report 3 cases of AVLC. The summary of the clinical features of our serie is shown in table l. Ages ranged from 46 to $72 \mathrm{yr}$ (average $60 \mathrm{yr}$ ). Two cases were presented after gynecologic cancer treatment (surgery, lymphadenectomy, and radiotherapy) (Figs. 1A and B). One case was related to lower limb chronic lymphedema
(Fig. 1C). The average range of the lapse of time between the onset of the predisposing factor and the LC onset was 6 yr (ranging between 2 and $10 \mathrm{yr}$ ). In our patients, LC presented with the same lesions: they had several small verrucous papules with intervening normal skin (Figs. 1A - C). A careful dermoscopic examination of the lesions showed the following features: lacunae, vascular structures and white lines. All lesions had a light background colour (Fig. 2). The initial clinical diagnosis in 2 of the cases was condyloma and were treated by electrocoagulation and trichloacetic acid, without improvement. Histopathologic findings were similar in all biopsy specimens. All lesions showed the presence of variably dilated vessels located within the superficial papillary dermis. These were lined by a single layer of endothelial cells with flat nuclei. The dermis between them did not show the presence of inflammation (Fig. 3). Morphologic features suggesting infection by human papillomavirus or dysplasia were not found. two patients were treated by Laser $\mathrm{CO} 2$ and cryotherapy, with a good response. The third patient refused a treatement and a conservative treatment was performed.

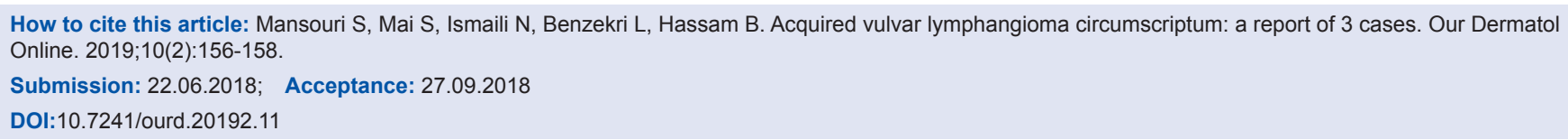




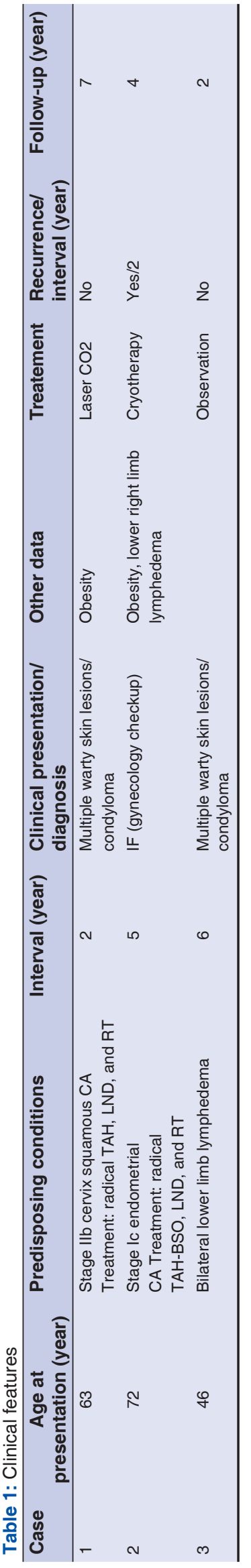

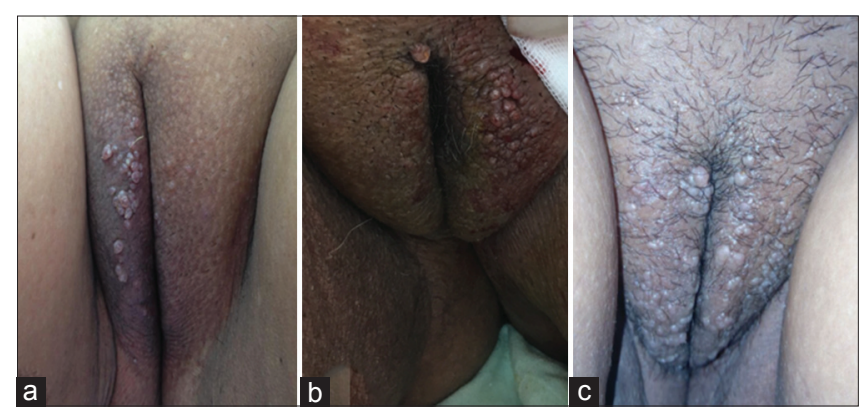

Figure 1: A and B: AVLC after gynecologic cancer treatment. C: AVLC related to lower limb chronic lymphedema

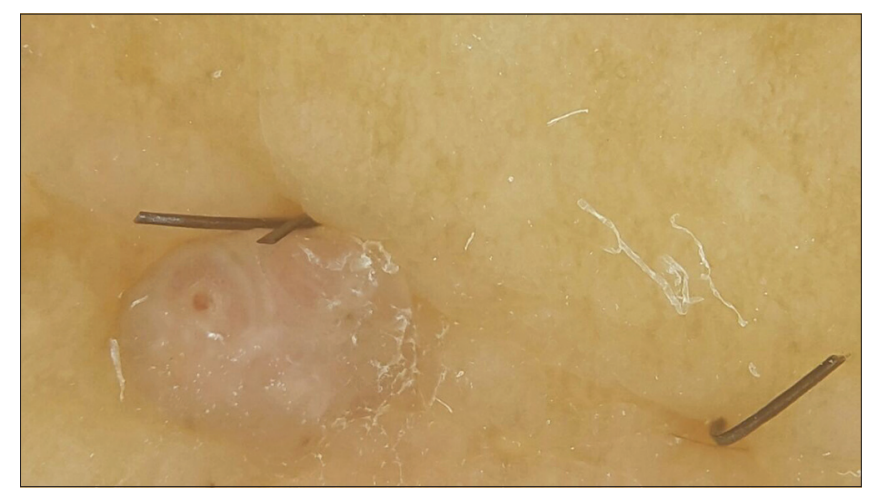

Figure 2: The dermoscopic view shows a yellow lacunae, white lines and vascular structures

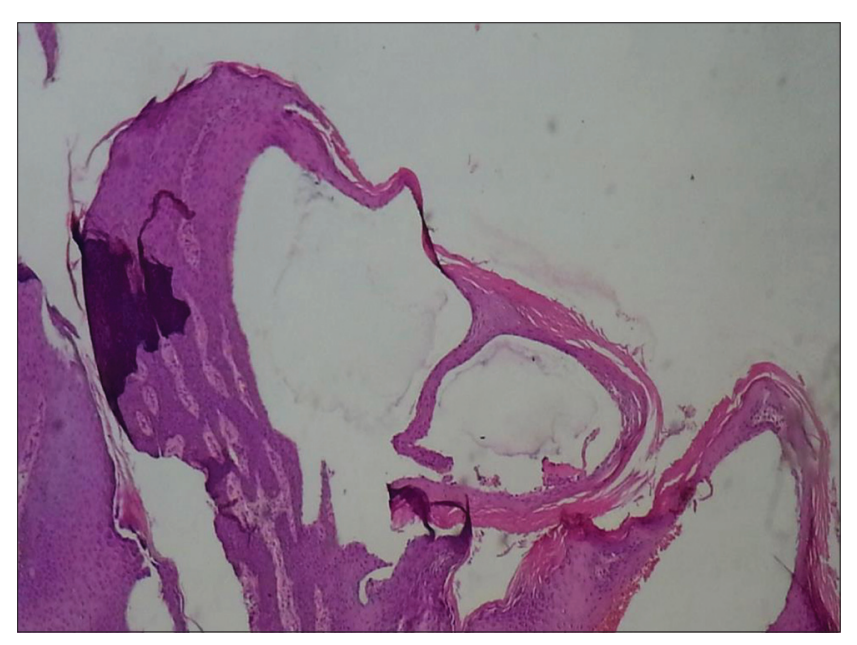

Figure 3: Histopathologic examination revealed the presence of dilated lymphatic vessels in the papillary dermis, bulging up the epidermis

Prior to the study, patient gave written consent to the examination and biopsy after having been informed about the procedure.

\section{DISCUSSION}

The AVLC, is a rare entity, as evidenced by less than 100 cases in the literature. Median age at diagnosis for acquired VLC is 52 years [4]. The lapse of time between 
the onset of the predisposing factor and the presentation of the LC is usually prolonged, from 3 to 40 years.

The AVLC is a long-term complication caused by different etiologies [5]. In most cases, the AVLC are associated to malignancy. It usually arises after radical surgery or cervical cancer radiotherapy. However, there have also been cases after treatment for vulvar cancer, endometrial cancer, or melanoma [3]. A large obstructive pelvic malignancy could lead to VLC (4). It is less often found in non oncological etiologies such as: Crohn disease [5], genital tuberculosis [6], chronic lymphedema [7], or local infectious diseases (acute cellulitis or hidradenitis suppurativa) [8]. Obesity could also have played a role in the development of LC. Several cases in the literature are without known cause [9].

The clinical presentation of ALV is highly variable, ranging from being asymptomatic to a highly disabling condition. Discomfort, itching, rubbing and lymph oozing are the most frequent symptoms [10]. Clinically, ALV usually presents as multiple nodular lesions, verruciform or polypoid, with variable size and, usually, with intervening normal skin. VLC sometimes accompanies lower extremity edema, or genital lymphedema. VLC may pose diagnostic difficulties both clinically and histopathologically. ALV is frequently confounded with warts or condyloma acuminata [11]. The most common dermoscopic pattern associated with ALV is the presence of lacunae and vascular structures. The hypopyon sign and the white or yellowish coloration of lacunae are very characteristic of lymphangioma circumscriptum [12]. Histopathologically, LC is characterized by the presence of dilated lymphatic channels in the papillary dermis. They have a cystic appearance and are layered by a normalappearing endothelium. The overlying epidermis can show variable degrees of acanthosis and hyperkeratosis [9].

The management of vulvar LC is not standardized and many different treatments with varying results have been proposed. Therapeutic options include surgical excision, yttrium-aluminium-garnet laser, cryotherapy, sclerotherapy, CO2 laser, or electrocoagulation. Conservative treatment is suitable in some cases and, probably, It's the best choice in asymptomatic cases, because of the high recurrence rate in all modalities of treatment [4].

\section{CONCLUSION}

VLC is a very rare disease, causing major discomfort, aesthetic prejudice and risk of infection. it is important to make clinicians aware of this entity so that it can be recognized more rapidly, and to prevent misdiagnosis and unnecessary treatments. when vulvar condylomas or warts are refractory to medical treatment, performing a biopsy should be considered in order to confirm the histology of the condition.

\section{CONSENT}

The examination of the patient was conducted according to the Declaration of Helsinki principles.

\section{REFERENCES}

1. LeBoit PE, Burg G, Weedon D, et al, eds. World Health Organization Classification of Tumours. Pathology and Genetics of Skin Tumours. Lyon: IARC Press; 2006:247-9.

2. Sangu " enza OP, Requena L, eds. Pathology of Vascular Skin Lesions: Clinicopathologic Correlations. Totowa, NJ: Humana Press; 2003.

3. Stewart CJ, Chan T, Platten M. Acquired lymphangiectasia (lymphangioma circumscriptum) of the vulva: a report of eight cases. Pathology. 2009;41:448-53.

4. Chang MB, Newman CC, Davis MDP, Lehman JS. Acquired lymphangiectasia (lymphangioma circumscriptum) of the vulva: Clinicopathologic study of 11 patients from a single institution and 67 from the literature. Int J Dermatol. 2016;55:e482-7.

5. Papalas JA, Sangueza OP, Puri PK, Robboy SJ, Selim MA. Vulvar vascular tumors: a clinicopathologic study of 85 patients. Am J Dermatopathol. 2013;35:1-7; quiz 8-10.

6. Vlastos A-T, Malpica A, Follen M. Lymphangioma circum- scriptum of the vulva: a review of the literature. Obstet Gynecol. 2003;101:946-54.

7. Fadare O, Brannan SM, Arin-Silasi D, Parkash V. Localized lymphedema of the vulva: a clinicopathologic study of 2 cases and a review of the literature. Int J Gynecol Pathol. 2011;30:306-13.

8. Sims SM, McLean FW, Davis JD, Morgan LS, Wilkinson EJ. Vulvar lymphangioma circumscriptum: a report of 3 cases, 2 associated with vulvar carcinoma and 1 with hidradenitis suppurativa. J Low Genit Tract Dis. 2010;14:234-7.

9. Kokcu A, Sari S, Kefeli M. Primary vulvar lymphangioma circumscriptum: a case report and review of literature. J Low Genit Tract Dis. 2015;19:e1-5.

10. Simon L, Tre vidic P, Denis P, Vignes S. Vulvar lymphangioma circumscriptum: comparison of primary and acquired forms in a cohort of 57 patients. JEADV. 2018;32:e41-e85.

11. Sah SP, Yadav R, Rani S. Lymphangioma circumscriptum of the vulva mimicking genital wart: a case report and review of literature. J Obstet Gynaecol Res. 2001;27:293-6.

12. Zaballos P del Pozo LJ, Argenziano G, Karaarslan IK, Landi C, Vera A, et al. Dermoscopy of lymphangioma circumscriptum: A morphological study of 45 cases. Australas J Dermatol. 2018;59:e189-93.

Copyright by Siham Mansouri, et al. This is an open-access article distributed under the terms of the Creative Commons Attribution License, which permits unrestricted use, distribution, and reproduction in any medium, provided the original author and source are credited. Source of Support: Nil, Conflict of Interest: None declared. 